\title{
Systemic intraoperative lidocaine infusion for postoperative pain management in obese patients: $A$ randomized, placebo controlled pilot study
}

\author{
Emily N. Alvey ${ }^{1}$, Yiu-Hei Ching ${ }^{2}$, Rachel A. Karlnoski ${ }^{2}$, Prachiti H. Dalvi ${ }^{3}$, Michel M. Murr², Devanand Mangar ${ }^{3}$, \\ Enrico M. Camporesi*2,3 \\ ${ }^{1}$ Saint Louis University School of Medicine, St. Louis, Missouri, United States \\ ${ }^{2}$ University of South Florida Morsani College of Medicine, Tampa, Florida, United States \\ ${ }^{3}$ TEAMHealth Anesthesia, Tampa General Hospital, Tampa, Florida, United States
}

Received: February 25, 2016

DOI: $10.5430 /$ css.v2n4p10

\author{
Accepted: May 18, 2016 \\ Online Published: July 25, 2016 \\ URL: http://dx.doi.org/10.5430/css.v2n4p10
}

\begin{abstract}
Background: Systemic intraoperative lidocaine has been seen to decrease postoperative pain following abdominal surgery, but few studies have examined its use in morbidly obese patients.

Objective: We sought to evaluate the safety and effects of intravenous lidocaine in obese patients undergoing laparoscopic Roux-en-Y gastric bypass (RYGB) surgery.

Methods: Setting: University-affiliated, private hospital, United States. In this preliminary prospective, double-blinded, and placebo-controlled safety study, we enrolled 21 patients and 20 completed the study. Ten patients were randomly assigned to the study group and received a continuous infusion of lidocaine intravenously from induction of general anesthesia until the end of the operation. Ten other patients were assigned to the control group and received a dextrose placebo intravenously over the same time period. Subjects were followed for 24 hours postoperatively. The primary endpoint was the presence of symptoms of lidocaine toxicity at 1 hour postoperatively. Exploratory outcomes were adverse events, visual analog scale (VAS) pain scores, opioid consumption, time to first passage of flatus and bowel movement, and length of hospital stay.

Results: No significant differences were seen between groups for overt signs of lidocaine toxicity or adverse events, opioid consumption, postoperative pain scores, time to first passage of flatus or first bowel movement, or length of hospital. We identified a trend towards less opioid consumption in patients receiving lidocaine. We were underpowered to detect statistical differences due to our pilot study design; addressing safety as the primary outcome measure as opposed to efficacy.

Conclusions: In this study, intravenous lidocaine in RYGB surgery was found to be safe without differences in postoperative pain or adverse effects.
\end{abstract}

Key Words: Lidocaine, Postoperative pain, Analgesia, Bariatric surgery, Recovery

\section{INTRODUCTION}

The perioperative care of obese patients is challenging for anesthesiologists and surgeons due to their high risk of respiratory depression and hemodynamic compromise with opioid use. ${ }^{[1]}$ Specialized anesthetic management of these patients is necessary and postoperative pain management strategies should focus on adequate pain relief while mitigating the risk of complications. Alvarez et al. recommend multi-

\footnotetext{
*Correspondence: Enrico M. Camporesi, Prof.; Email: ecampore@health.usf.edu; Address: TEAMHealth Anesthesia, 1 Tampa General Circle, Suite A327, Tampa, Florida 33606, United States.
} 
modal pharmacological approaches for providing optimal analgesia after abdominal surgery. ${ }^{[2]} \mathrm{A}$ number of drugs and techniques have been used in an attempt to decrease postoperative pain while minimizing opioid use, including ketorolac, dexmedetomidine, ketamine, peripheral nerve blocks, neuraxial blocks, continuous delivery of local anesthetic using an infusion pump system, and intraperitoneal infusions, with varying results. ${ }^{[3-8]}$ Still, there is limited literature on signs and symptoms associated with local anesthetic toxicity, especially in obese patients.

Intraoperative intravenous lidocaine has been shown to reduce the incidence and severity of postoperative pain following abdominal surgery in non-obese patients; decrease postoperative opioid consumption, pain during mobilizing and coughing, and abdominal discomfort; be safe and welltolerated; and be associated with earlier return of bowel function and shorter hospital length of stay. ${ }^{[9-13]}$ Similarly, we hypothesized that intraoperative intravenous lidocaine could be used safely and would significantly decrease postoperative pain, narcotic requirements, time to flatus and bowel movement, and length of hospital stay compared to placebo in a group of obese patients. Therefore, we conducted a randomized pilot study comparing the safety and effects of intravenous lidocaine versus placebo in patients undergoing laparoscopic Roux-en-Y gastric bypass (RYGB) surgery.

\section{Materials AND Methods}

\subsection{Study design}

In this preliminary randomized, double-blinded, and placebo-controlled pilot study (ClinicalTrials.gov Identifier: NCT01944098), we compared intravenous lidocaine versus placebo in patients undergoing laparoscopic RYGB surgery. The study was conducted at a single facility (Tampa General Hospital). Approval for the study was obtained from the University of South Florida Institutional Review Board, and written informed consent was obtained from all study participants.

\subsection{Patients and randomization}

Patients scheduled to undergo laparoscopic RYGB surgery were assessed for eligibility during the preoperative anesthesia clinic evaluation, and eligible patients provided written informed consent at this time. Patients between the ages of 18-65 with a body mass index (BMI) greater than $40 \mathrm{~kg} / \mathrm{m}^{2}$ and less than $60 \mathrm{~kg} / \mathrm{m}^{2}$ were eligible for the study. Patients who were pregnant; had a history of chronic narcotic use, cirrhosis, portal hypertension, extensive intraperitoneal adhesions; reported allergies to lidocaine, hydromorphone, ketorolac, amide anesthetics, or corn; or found to have abnormal serum potassium, magnesium, alanine aminotransferase, and aspartate transaminase levels were excluded.

Randomization occurred using a computer-generated list by a biostatistician who then provided the patient assignments directly to an unblinded pharmacist. The remaining members of the team remained blinded. Of the 21 patients enrolled, 10 patients were assigned to the study group, 10 patients were assigned to the control group, and 1 patient decided not to participate after being enrolled.

\subsection{Study treatment}

For all patients, induction of general anesthesia was performed with propofol and a neuromuscular blocking agent. After adequate ventilation was confirmed, patients were started on an intravenous lidocaine or a placebo infusion at 2 milligrams per kilogram per hour $(\mathrm{mg} / \mathrm{kg} / \mathrm{hr})$ and continued through the end of the operation. Calculations were made using total body weight. General anesthesia was maintained with discretionary use of fentanyl, desflurane, midazolam, and dexmedetomidine per our institution's standard practice. After stopping the study drug infusion, all patients received intermittent morphine boluses during emergence at the discretion of the anesthesiologist. The laparoscopic RYGB operation was performed by a single, experienced bariatric surgeon (MMM) per standard of practice.

Postoperative pain management included a standing dose of ketorolac $60 \mathrm{mg}$ in the post-anesthesia care unit (PACU; $30 \mathrm{mg}$ intravenous and $30 \mathrm{mg}$ intramuscular), then $15 \mathrm{mg}$ intravenous scheduled every 6 hours for 24 hours. Hydromorphone was added via patient-controlled analgesia at a demand dose of $0.2 \mathrm{mg}$ every 10 minutes without a basal rate. The total amount of opioids used within 24 hours after the procedure was calculated as intravenous morphine milligram equivalents.

\subsection{Outcome measures}

The primary outcome was the presence of lidocaine toxicity symptoms at 1 hour after lidocaine infusion cessation. Patients were asked of any symptoms of lidocaine toxicity an hour after entering PACU (1 hour is the average time for a patient undergoing a laparoscopic RYGB to become lucid after anesthesia) using a questionnaire to assess symptoms of lightheadedness, numbness around tongue or mouth, dizziness or metallic taste in mouth. Blood pressure was also assessed and compared to baseline (pre-surgery). Blood pressure elevations of greater than $20 \%$ from baseline were considered significant.

The secondary outcomes were pain at rest, coughing, and moving at 6,12,18 and 24 hours postoperatively using the VAS (0 $\mathrm{cm}$ as no pain $-10 \mathrm{~cm}$ as maximum pain), total opioid consumption, time to the first passage of flatus and 
the first bowel movement, and length of hospital stay.

\subsection{Statistical analysis}

Sample size selection was based on the feasibility of completing a small number of subjects in a pilot study to be used in the development of a larger study including sample size determination with power analysis. Data were analyzed using SPSS 20.0 (SPSS Inc, IL). Distributions were examined for normality using visual inspection of box plots and the Shapiro-Wilk test of normality. Group comparisons were conducted using Fisher Exact and Chi-Square for categorical variables and Mann-Whitney $U$ and t-test for continuous variables. We performed the Fisher exact test to determine the primary outcome (lidocaine toxicity at 1 hour). We used the Wilcoxon rank-sum test to evaluate the differences in VAS scores at each time point, as well as the mixed-effects model adjusted for treatment by time interaction to determine the differences in VAS scores between the two treatment groups over time. Results are expressed as mean $\pm S D$ for normally distributed values or median for nonparametric values. A $p$-value of $<.05$ was considered statistically significant. Data were analyzed in a blinded fashion, group assignments were revealed by the study pharmacist after analysis was completed.

\section{RESUlts}

\subsection{Patients}

Between August 2013 and May 2014, 58 patients were assessed for eligibility. Thirty-one patients did not meet inclusion criteria, and 7 patients met the inclusion criteria but did not agree to participate in the study (see Figure 1). Twentyone agreed to participate, but 1 subject was excluded after signing the consent form as a result of abnormal preoperative laboratory testing. Twenty subjects were randomized, with 10 receiving intravenous lidocaine and 10 receiving placebo. Of the 20 subjects, 19 completed the study. In one subject, drug administration was stopped after 109 minutes into a total 206-minute operation after portal hypertension was discovered intraoperatively. No significant differences were present between the demographics or baseline characteristics between the two groups (see Table 1).

\subsection{Lidocaine toxicity}

No significant differences were seen for symptoms of lidocaine toxicity between study groups (see Table 2). In the lidocaine group, no subjects reported lightheadedness or dizziness, 1 reported numbness around the mouth, and 4 reported a metallic taste in the mouth. In the placebo group, 1 patient reported lightheadedness, 3 dizziness, 1 numbness around the mouth, and 1 a metallic taste in the mouth. Lidocaine infusion did not have a significant effect on blood pressures $(120.4 \pm 23.5 \mathrm{mmHg} / 62.6 \pm 12.74 \mathrm{mmHg}$ in the lidocaine group and $117.5 \pm 17.9 \mathrm{mmHg} / 69.5 \pm 9.56 \mathrm{mmHg}$ in the placebo group).

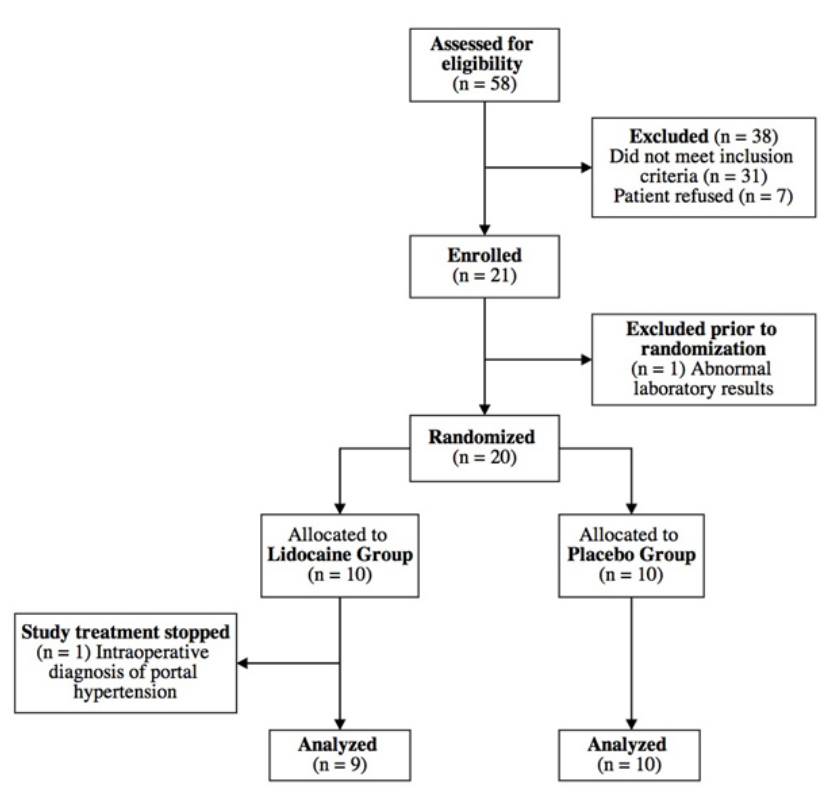

Figure 1. CONSORT flow study diagram

\subsection{Postoperative pain and opioid consumption}

Pain scores between the two groups were similar, with no significant differences between the two groups (see Table 3). At 6 hours after the end of the procedure, the average resting VAS pain score was marginally lower for the lidocaine group (3 [0-10]) than for the placebo group (4.5 [0-8], $p=.07)$, while the coughing (8 [3-10] vs. 8 [5-9], $p=1.00)$ and moving (8 [0-10] vs. 8 [4-9], $p=1.00)$ scores were similar for the two groups. At 12 hours, the resting (3.5 [0-9] vs. 4 [0-6], $p=1.00)$, coughing (7 [5-10] vs. 7 [2-9], $p=.65)$, and moving ( 8 [5-10] vs. 7 [5-8], $p=.27$ ) VAS pain scores were again similar for the lidocaine and placebo groups. At 18 hours, the lidocaine group reported slightly lower resting pain scores (2.5 [0-9]) than the placebo group (4.5 [0-8], $p=.37)$. The coughing $(6$ [2-10] vs. 6.5 [4-9], $p=1.00)$ and moving (6 [2-10] vs. 7 [4-8], $p=1.00)$ pain scores were again similar for the two groups. At 24 hours, the average resting pain score was slightly lower for the lidocaine group (3 [0-8]) than for the placebo group (3.5 [0-10], $p=.65)$. The average coughing (8 [2-10] vs. 5.5 [4-10], $p=.65)$ and moving (6 [2-10] vs. 4.5 [4-10], $p=.64)$ pain scores were slightly higher for the lidocaine group than the placebo group; however, none of the differences in pain scores between the two groups was statistically significant (see Figure 2). There was no difference in the overall change in VAS resting scores $(p=.347)$, VAS coughing scores $(p=.606)$, or VAS mobile scores $(p=.626)$ between groups. 
Table 1. Patient demographics and characteristics

\begin{tabular}{lllc}
\hline & Lidocaine $(\mathbf{n}=\mathbf{1 0})$ & Placebo $(\mathbf{n}=\mathbf{1 0})$ & $\boldsymbol{p}$-value \\
\hline Age & $47.3 \pm 8.3$ & $47.5 \pm 7.4$ & .940 \\
BMI $\left(\mathrm{kg} / \mathrm{m}^{2}\right)$ & $46.4 \pm 6.1$ & $48.3 \pm 5.6$ & .496 \\
Gender & & & .628 \\
$\quad$ - Female & 8 & 6 & \\
- Male & 2 & 4 & .057 \\
Obstructive sleep apnea & & & \\
- No & 6 & 1 & .256 \\
- Yes & 4 & 9 & .384 \\
Total study drug infusion volume (ml) & $185.8 \pm 65.1$ & $218.1 \pm 60.5$ & .879 \\
Infusion time (minutes) & $166.4 \pm 45.9$ & $184.1 \pm 40.3$ & N/A \\
Total surgery time (minutes) & $162.6 \pm 32.6$ & $162.4 \pm 40.8$ & \\
Total amount of lidocaine infused (mg) & $767.1 \pm 239.3$ & 0 & \\
\hline
\end{tabular}

Note. BMI: body mass index

Table 2. Symptoms of lidocaine toxicity

\begin{tabular}{llll}
\hline & Lidocaine $(\mathbf{n}=\mathbf{1 0})$ & Placebo $(\mathbf{n}=\mathbf{1 0})$ & p-value \\
\hline $\begin{array}{l}\text { Lightheadedness } \\
\text { - No }\end{array}$ & 10 & 9 & 1.000 \\
$\begin{array}{l}\text { - Yes } \\
\text { Dizziness }\end{array}$ & 0 & 1 & .211 \\
- No & 10 & & \\
- Yes & 0 & 7 & 1.000 \\
Mouth Numbness & & 3 & \\
- No & 9 & 9 & .303 \\
- Yes & 1 & 1 & \\
Metallic Taste & & & .677 \\
- No & 6 & 9 & .172 \\
- Yes & 4 & 1 & $117.5 \pm 17.9$ \\
Post-op Systolic (mmHg) & $120.4 \pm 23.5$ & $69.5 \pm 9.56$ & \\
Post-op Diastolic (mmHg) & $62.6 \pm 12.74$ & & \\
\hline
\end{tabular}

Note. mmHg: millimeter of mercury

The mean number of morphine equivalents consumed during the first 24 hours was slightly lower for the lidocaine group $(52.1 \pm 35.6$ morphine mg equivalents) than for the placebo group ( $68.3 \pm 27.5$ morphine mg equivalents, $p=.253)$, but the difference was not statistically significant.

\subsection{Return of flatus and bowel function and length of hospital stay}

The time until first passage of flatus and time until first bowel movement were also similar for the lidocaine and placebo groups (63.1 \pm 19 vs. $48.2 \pm 19$ hours, $p=.149$, and $62.4 \pm 18.5$ vs. $60 \pm 16.1$ hours, $p=.713$, respectively). The length of hospital stay was similar for the two groups $(4.8 \pm 1.9$ days in the lidocaine group vs. $4.7 \pm 1.6$ days in the placebo group, $p=.907)$.

Published by Sciedu Press

\section{Discussion}

Intravenous lidocaine infusion perioperatively has been used to manage postoperative pain and recovery for more than 20 years; thus, there is extensive literature supporting this practice. ${ }^{[14]}$ Still, our understanding of lidocaine infusion in morbidly obese patients remains underdeveloped. Our current report illustrates the safety of lidocaine infusion in adult bariatric patients with a pilot cohort of twenty patients. In doing so, it lays the foundation for a future, randomized clinical trial.

Several studies, especially in abdominal surgery, have evaluated lidocaine toxicity for various time points post-lidocaine infusion and have proved to be effective. Contrastingly, spinal surgeries and thoracotomies have not been noted to achieve the same results with lidocaine infusion. Because of lidocaine's efficacy in decreasing post-operative pain, it may 
have an opioid sparing effect: a finding that is significant since morbidly obese patients undergoing bariatric surgery are at a high risk of post-operative depression. Though the $p$ value was not significant $(p$-value $\leq .05)$, the lidocaine group consumed less morphine in the first 24 hours on average than the placebo group. Perhaps a larger sample size would pro- vide more conclusive results regarding lidocaine infusion's morphine sparing effect. Additionally, only one patient experienced overt cardiac arrhythmia toxicity attributable to lidocaine infusion. A few other patients in Table 2 had milder symptoms of toxicity.

Table 3. Postoperative data

\begin{tabular}{|c|c|c|c|}
\hline & Lidocaine $(n=9)$ & Placebo $(n=10)$ & $p$-value \\
\hline \multicolumn{4}{|l|}{ VAS score at 6 hours post-op } \\
\hline • Resting & $3(0-10)$ & $4.5(0-8)$ & .070 \\
\hline - Coughing & $8(3-10)$ & $8(5-9)$ & 1.000 \\
\hline - Moving & $8(0-10)$ & $8(4-9)$ & 1.000 \\
\hline \multicolumn{4}{|l|}{ VAS score at 12 hours post-op } \\
\hline • Resting & $3.5(0-9)$ & $4(0-6)$ & 1.000 \\
\hline - Coughing & $7(5-10)$ & $7(2-9)$ & .650 \\
\hline - Moving & $8(5-10)$ & $7(5-8)$ & .270 \\
\hline \multicolumn{4}{|l|}{ VAS score at 18 hours post-op } \\
\hline • Resting & $2.5(0-9)$ & $4.5(0-8)$ & .370 \\
\hline - Coughing & $6(2-10)$ & $6.5(4-9)$ & 1.000 \\
\hline - Moving & $6(4-9)$ & $7(4-8)$ & 1.000 \\
\hline \multicolumn{4}{|l|}{ VAS score at 24 hours post-op } \\
\hline • Resting & $3(0-8)$ & $3.5(0-10)$ & .650 \\
\hline - Coughing & $8(2-10)$ & $5.5(4-10)$ & .650 \\
\hline - Moving & $6(2-10)$ & $4.5(4-10)$ & .640 \\
\hline Opioids (morphine mg equivalents) & $52.1 \pm 35.6$ & $68.3 \pm 27.5$ & .253 \\
\hline Time to first passage of flatus (hours) & $63.1 \pm 19$ & $48.2 \pm 19$ & .149 \\
\hline Time to first bowel movement (hours) & $62.4 \pm 18.5$ & $60 \pm 16.1$ & .713 \\
\hline Length of hospital stay (days) & $4.8 \pm 1.9$ & $4.7 \pm 1.6$ & .907 \\
\hline
\end{tabular}

Note. VAS: visual analog scale

Toxic levels of lidocaine first present as subclinical symptoms before reaching clinical manifestations: the literature reports perioral numbness, tingling in fingers and elsewhere before higher pretoxic level of lidocaine. We developed an assessment based upon the subclinical symptoms to evaluate our primary safety outcome. In our study, subjects who received lidocaine did not report increased symptoms of lidocaine toxicity compared to subjects who received placebo. Additionally, hypertension was measured as an objective outcome of lidocaine toxicity. Patients in the lidocaine group did not have significantly higher blood pressures relative to the placebo group. Neither the lidocaine nor the placebo group showed postoperative hypertension, indicating that pain was adequately controlled after the procedure. Neither group experienced post-operative cardiovascular or neurological adverse events. From these assessments, we concluded that intravenous lidocaine was safe to administer intraoperatively during RYGB surgery.

Lidocaine infusion trials involving intra-abdominal surgery demonstrated a significant reduction in total analgesic intake in the postoperative period up to 48 to 72 hours. ${ }^{[9-13,15-17]}$ The reductions in analgesic requirements were between $33 \%$ to $35 \%$ when lidocaine infusion was maintained for 0-1 hour postoperatively, and up to $83 \%$ compared with placebo group when lidocaine infusion was continued for 24 hours postoperatively. ${ }^{[15]}$ In our report, the first VAS score was obtained at 6 hours post-op, possibly when the effect of lidocaine was dissipated and the small differences between lidocaine and placebo group do not reach significance. The Kaba et al. study involving laparoscopic colectomy further indicated that lidocaine infusion maintained for 24 hours postoperatively was more effective in reducing pain during mobilization and when coughing than at rest for 0 to 24 hours, while abdominal discomfort was also significantly reduced by lidocaine. ${ }^{[9]}$

De Oliveira et al. published a similar study, powered to measure quality of recovery, and reported that continuous intravenous lidocaine infusion during bariatric surgery decreased opioid consumption and improved the patients' quality of 
recovery in morbidly obese patients (BMI $\left.42-54 \mathrm{~kg} / \mathrm{m}^{2}\right){ }^{[15]}$ The results of our pilot study also suggest superiority of lidocaine infusion for immediate post-operative pain control when compared to placebo. Subjects who received intravenous lidocaine reported slightly lower pain scores, on average, than subjects who received placebo, but the differ-

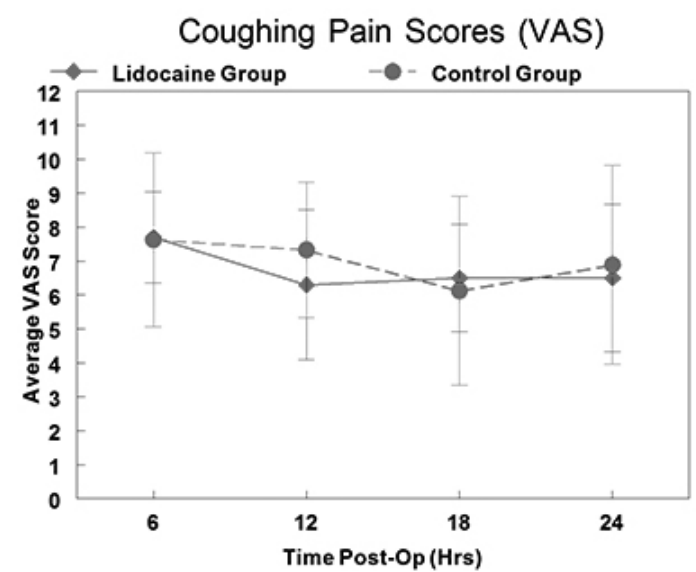

ence did not reach statistical significance. Additionally, we observed a trend toward decreased consumption of narcotic analgesics in lidocaine patients, but the difference was again not statistically significant. The sample size for this study was limited and did not provide our study with adequate power to detect statistically significant differences.

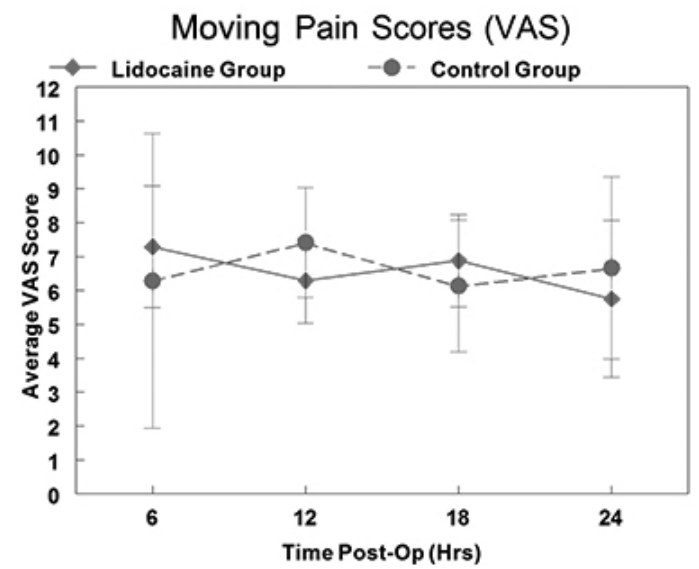

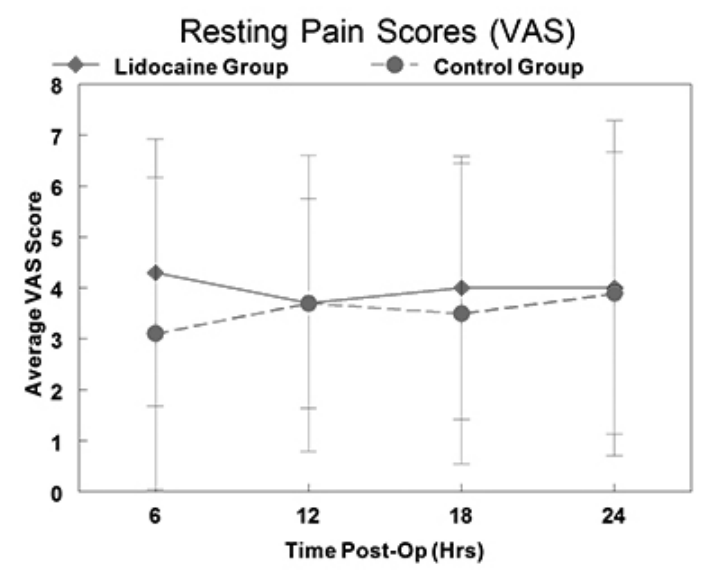

Figure 2. Postoperative VAS pain scores

The mean coughing and moving pain scores were slightly higher for the lidocaine group than the placebo group, but no statistically significant differences were seen in the VAS pain scores between groups

Several trials that investigated the impact of intravenous lidocaine infusion on postoperative analgesia and recovery from abdominal surgery also showed that lidocaine significantly expedited return of bowel function. ${ }^{[9-13,15-17]}$ The first passage of flatus occurred 8 to 24 hours earlier and the first bowel movement occurred 12 to 28 hours earlier in lidocaine-treated patients compared with placebo recipients. ${ }^{[12]}$ Rimback et al. reported significantly earlier return of propulsive motility in the colon in the lidocaine-treated group after cholecystectomy. ${ }^{[10]}$ Another study reported that time until which patients could tolerate solid foods was notably shorter in lidocaine-treated patients even though lidocaine was terminated at 4 hours postoperative. ${ }^{[17]}$ In patients having laparoscopic colectomies, where lidocaine was administered for 24 hours postoperative, first flatus was noted to return 12 hours earlier and defecation returned 24 hours earlier in lidocaine-treated patients. ${ }^{[9]}$ Our results did not show any differences in the time to first passage of flatus or time to first bowel movement. However, our study was not powered to measure this outcome.

Length of hospital stay was also shown to be significantly reduced for lidocaine-treated patients. ${ }^{[9,12,13]}$ The time to hospital discharge was an average of 1.1 days shorter in the lidocaine group even though lidocaine infusion was terminated 1 hour postoperatively. ${ }^{[13]}$ The Kaba et al. study, where lidocaine infusion was continued 24 hours postopera- 
tively, also showed that hospital stay was 1 day shorter in the

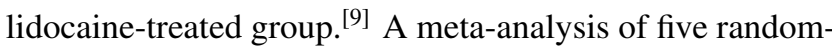
ized controlled trials including 220 patients also reported that lidocaine-treated patients undergoing open or laparoscopic abdominal surgery have shorter length of hospital stay than the control groups. ${ }^{[12]}$ However, De Oliveira et al. reported no difference in time to meet hospital discharge between the lidocaine group and the saline control group after bariatric surgery. We also did not find a difference in length of stay. ${ }^{[15]}$

This prospective trial has limitations regarding sample size and patient selection as it was designed to serve as a pilot study to assess safety with the intent to complete a larger randomized, prospective trial. Based upon evaluation of subclinical symptoms, we found that intraoperative infusions in the bariatric population are safe. A follow-up study with a larger cohort would help us establish efficacy of lidocaine in managing post-operative pain in obese patients and further build upon the results of De Olivera and colleagues. ${ }^{[15]}$

\section{Conclusions}

Our pilot study documents that a continuous intraoperative lidocaine infusion in adult bariatric patients with BMI ranging from $40 \mathrm{~kg} / \mathrm{m}^{2}$ to $60 \mathrm{~kg} / \mathrm{m}^{2}$ can be safely undertaken at the infusion rate of $2 \mathrm{mg} / \mathrm{kg} / \mathrm{hr}$. Our study suggests the need for a larger prospective study in which we would start lidocaine infusion at the end of the surgical procedure and maintain it for some time post-operatively.

\section{CONFLICTS OF INTEREST Disclosure}

The authors have no conflicts of interest to disclose.

\section{REFERENCES}

[1] Wadhwa A, Singh PM, Sinha AC. Airway management in patients with morbid obesity. International Anesthesiology Clinics. 2013 JunAug; 51(3): 26-40. PMid: 23797643. http://dx.doi.org/10.10 97/AIA. Ob013e318298140f

[2] Alvarez A, Singh PM, Sinha AC. Postoperative analgesia in morbid obesity. Obes Surg. 2014 Jan 16; 24: 652-9. PMid: 24431032 http://dx.doi.org/10.1007/s11695-014-1185-2

[3] Govindarajan R, Ghosh B, Sathyamoorthy MK, et al. Efficacy of ketorolac in lieu of narcotics in the operative management of laparoscopic surgery for morbid obesity. Surg Obes Relat Dis. 2005 Nov-Dec; 1(6): 530-535. PMid: 16925285. http://dx.doi.org $/ 10.1016 / \mathrm{j}$. soard.2005.08.013

[4] Tufanogullari B, White PF, Peixoto MP, et al. Dexmedetomidine infusion during laparoscopic bariatric surgery: the effect on recovery outcome variables. Anesth Analg. 2008 Jun; 106(6): 1741-8. PMid: 18499604.http://dx.doi.org/10.1213/ane.0b013e3 $18172 \mathrm{c} 47 \mathrm{c}$

[5] Zakine J, Samarcq D, Lorne E, et al. Postoperative ketamine administration decreases morphine consumption in major abdominal surgery: a prospective, randomized, double-blind, controlled study. Anesth Analg. 2008 Jun; 106(6): 1856-61. PMid: 18499623 http://dx.doi.org/10.1213/ane.0b013e3181732776

[6] Cohen AR, Smith AN, Henriksen BS. Postoperative Opioid Requirements Following Roux-en-Y Gastric Bypass in Patients Receiving Continuous Bupivacaine Through a Pump System: A Retrospective Review. Hosp Pharm. 2013 May 29; 48(6): 479-83. PMid: 24421509. http://dx.doi.org/10.1310/hpj4806-479

[7] Medbery RL, Chiruvella A, Srinivasan J, et al. The value of continuous wound infusion systems for postoperative pain control following laparoscopic Roux-en-Y gastric bypass: an analysis of outcomes and cost. Obes Surg. 2014 Apr; 24(4): 541-8. PMid: 24421154. http: //dx.doi .org/10.1007/s11695-013-1110-0

[8] Ram D, Sistla SC, Karthikeyan VS, et al. Comparison of intravenous and intraperitoneal lignocaine for pain relief following laparoscopic cholecystectomy: a double-blind, randomized, clinical trial. Surg Endosc. 2014 Apr; 28(4): 1291-7. PMid: 24357420 http://dx.doi.org/10.1007/s00464-013-3325-5
[9] Kaba A, Laurent SR, Detroz BJ, et al. Intravenous lidocaine infusion facilitates acute rehabilitation after laparoscopic colectomy. Anesthesiology. 2007 Jan; 106(1): 11-8; discussion 5-6. PMid: 17197840. http://dx.doi.org/10.1097/00000542-200701000-00007

[10] Rimbäck G, Cassuto J, Tollesson PO. Treatment of postoperative paralytic ileus by intravenous lidocaine infusion. Anesth Analg. 1990 Apr; 70(4): 414-9. PMid: 2316883.

[11] Cassuto J, Wallin G, Högström S, et al. Inhibition of postoperative pain by continuous low-dose intravenous infusion of lidocaine. Anesth Analg. 1985 Oct; 64(10): 971-4. PMid: 3898920. http://dx.doi.org/10.1213/00000539-198510000-00005

[12] McCarthy GC, Megalla SA, Habib AS. Impact of intravenous lidocaine infusion on postoperative analgesia and recovery from surgery: a systematic review of randomized controlled trials. Drugs. 2010 Jun 18; 70(9): 1149-63. PMid: 20518581. http://dx.doi.org/10. 2165/10898560-000000000-00000

[13] Groudine SB, Fisher HA, Kaufman RP, et al. Intravenous lidocaine speeds the return of bowel function, decreases postoperative pain, and shortens hospital stay in patients undergoing radical retropubic prostatectomy. Anesth Analg. 1998 Feb; 86(2): 235-9. PMid: 9459225.

[14] Kranke P, Jokinen J, Pace NL, et al. Continuous intravenous perioperative lidocaine infusion for postoperative pain and recovery. Cochrane Database of Syst Rev. 2015 Jul 16; 7: CD009642. http://dx.doi.org/10.1002/14651858.cd009642.pub2

[15] De Oliveira GS, Duncan K, Fitzgerald P, et al. Systemic lidocaine to improve quality of recovery after laparoscopic bariatric surgery: a randomized double-blinded placebo-controlled trial. Obes Surg. 2014 Feb; 24(2): 212-8. PMid: 24036842. http://dx.doi.org/1 $0.1007 / \mathrm{s} 11695-013-1077-\mathrm{x}$

[16] Pellis T, Leykin Y, Albano G, et al. Perioperative management and monitoring of a super-obese patient. Obes Surg. 2004 Nov-Dec; 14(10): 1423-7. PMid: 15603664. http://dx.doi.org/10.1381 /0960892042583914

[17] Herroeder S, Pecher S, Schonherr ME, et al. Systemic lidocaine shortens length of hospital stay after colorectal surgery: a double-blinded, randomized, placebo-controlled trial. Ann Surg. 2007 Aug; 246(2): 192-200. PMid: 17667496. http://dx.doi.org/10.1097/SLA .0b013e31805dac11 